\title{
Why Introduce Machine Learning To Rural Health Care?
}

\author{
Alfred A. Cecchetti, PhD, MSc ${ }^{1}$
}

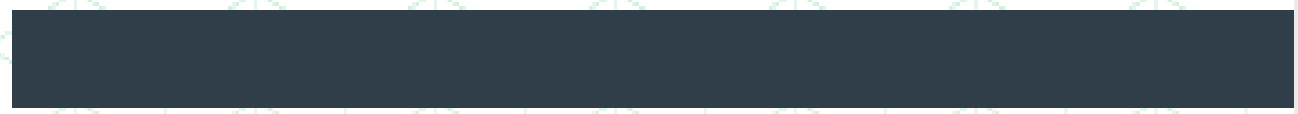

Author affiliations are

listed at the end of this

article.

Correspondence to:

Alfred A. Cecchetti, PhD

Marshall University

Joan C. Edwards

School of Medicine

cecchetti@marshall.edu

\section{KEYWORDS}

machine learning, rural health care

Machine learning, the process of teaching a machine to recognize patterns without explicitly being programmed, can utilize existing clinical data sets to detect patterns and trends and predict future medical outcome/events, thereby, giving us the ability to proactively influence patient health. It especially lends itself to those areas of healthcare that have reproducible or standardized processes such as care management.

Machine learning has three primary categories: supervised learning, unsupervised learning, and reinforcement learning. Supervised learning uses an expert (e.g., someone who has a strong understanding of that field) to label medical data with an outcome. The produced model then learns the relationship between the data and the outcome to produce future prediction on new data. Unsupervised learning does not use an expert to label its data with an outcome, but develops a model that sorts and separates that data into groups. The unsupervised model then can separate new data set into groups of its choice. Reinforcement learning uses trial and error to figure out an optimal goal. In this model, the training data are expected to provide only an indication as to whether an action is correct or not. An evaluation process on the above machine learning categories to determine how "correct" the developed models are can then be introduced and assessed.

\section{RURAL DISPARITIES}

Disparities exist in the health care system; rural populations show higher rates of chronic illness and poorer overall health. 1,2,3,4,5 Rural health has not received the same attention as the health problems found in the urban population.

The Centers for Disease Control and Prevention list the top ten causes of death in West Virginia in the following order: heart disease, cancer, accidents, chronic lower respiratory diseases, stroke, diabetes, alzheimer's disease, kidney disease, pneumonia, and suicide. ${ }^{6}$ For each of these causes of death a machine learning algorithm has been designed to predict outcomes: heart disease ${ }^{7,8}$, cancer ${ }^{9,10}$, accidents ${ }^{11,12}$, respiratory disease $\mathrm{e}^{13,14}$, stroke ${ }^{15,16}$, diabetes ${ }^{17,18}$, alzheimer's disease ${ }^{19,20}$, kidney disease ${ }^{21,22}$, pneumonia ${ }^{23,24}$, and suicide ${ }^{25,26}$. Machine learning can 
also help rural health in disease identification and management, diagnosis (especially when "diagnosis of elimination" is required), personalized medicine, behavioral issues, clinical trial research, radiology, radiotherapy, and epidemic outbreak prediction.

Machine learning has the potential to focus its predictive power on fast classification or even on prevention of rural health disparities at low cost and high speed.

\section{WHY FOCUS ON THE RURAL POPULATION?}

A look at rural populations shows that this group has special problems due to a higher mix of older individuals who have lower financial resources, educational deficiencies, and tendency to be more ill than urban populations. ${ }^{27}$ The physician to patients ratio is typically very inadequate in rural areas resulting in long and in many cases arduous travel distances to obtain health services, long wait times for appointments, and limited access to certain specialists within the rural geographic areas.

Rural populations can benefit in a number of ways by machine learning. Those medical specialties that use images (cardiology, radiology, pathology) can, through the use of machine learning algorithms, provide faster and cheaper services without the need for high cost specialists who can now spend more time dealing directly with their patients. Machine learning is already showing signs of promise using experimental novel techniques in mental healthcare. For instance, in the detection of changes in mental states for bipolar patients in a rural psychiatric hospital in Austria, a machine learning app for depression/mania recognition produced an accuracy of $72-81 \% .{ }^{28}$ This is very promising for rural populations, since these machine-learning approaches suggest that the ability to diagnosis dangerous medical conditions without the need to have a local and expensive specialized healthcare provider nearby is possible.

\section{MACHINE LEARNING OBSTACLES}

There are difficulties, as well, associated with the use of machine learning methods especially in the rural areas. Some methods use "black box" approaches where the actual reasoning of the model is difficult to discern. Data silos can be difficult to overcome. Technical support is often limited with few resources to allocate to this area. In urban areas, stricter controls on the insertion of data in the electronic medical records can be enforced. Rural areas tend to have less control on this process. Another major concern is bias (cultural, educational, gender, race, location, etc.) in the development of these algorithms. Regional differences found within the supervised machine learning models may need to be better understood to improve accuracy within these rural models.

\section{THE FUTURE OF MACHINE LEARNING IN RURAL HEALTH CARE}

Using advanced analytics, algorithms can assist rural medical providers by providing better information at lower cost. They have the potential to open the floodgates to the combined brainpower and expertise of the best top-notch healthcare providers and organizations for the rural population and thus provide them access to the best care they can get.

Machine learning and the associated algorithms they produce can be another powerful tool in the rural health care provider's toolbox to understand and predict disease and other medical events while lowering the cost of receiving these services.

\section{AUTHOR AFFILIATIONS}

1. Marshall University Joan C. Edwards School of Medicine, Huntington, West Virginia

\section{REFERENCES}

1. Rabinowitz HK, Paynter NP. The rural vs urban practice decision. Jama. 2001;287(1):113.

2. Anderson AE, Henry KA, Samadder NJ, Merrill RM, Kinney AY. Rural vs urban residence affects risk-appropriate colorectal cancer screening. Clinical Gastroenterology and Hepatology. 
2013;11(5):526-533.

3. Reif S, Whetten K, Ostermann J, Raper JL. Characteristics of HIV-infected adults in the deep south and their utilization of mental health services: a rural vs urban comparison. AIDS Care. 2006;18(S1):10-17.

4. Shubhakaran KP, Khichar RJ. Stroke management disparity in urban vs rural locations. ANN Publications. Neurology.

5. Newgard CD, Fu R, Bulger E, Hedges JR, Mann NC, Wright DA, Lehrfeld DP, Shields C, Hoskins G, Warden C, Wittwer L, Cook JN, Verkest M, Conway W, Somerville S, Hansen M. Evaluation of rural vs urban trauma patients served by 9-1-1 emergency medical services. JAMA Surgery. 2017;152(1):11-18.

6. Centers for Disease Control and Prevention (April 2018) National Center for Health Statistics, downloaded from https://www.cdc.gov/nchs/ pressroom/states/westvirginia/westvirginia.htm.

7. Sreejith S, Rahul S, Jisha RC. 2016. A real time patient monitoring system for heart disease prediction using random forest algorithm. In Advances in Signal Processing and Intelligent Recognition Systems (pp. 485-500). Springer, Cham.

8. Patil PR, Kinariwala SA. 2017. Automated Diagnosis of Heart Disease using Random Forest Algorithm. International Journal of Advance Research, Ideas and Innovations in Technology. https://github. com/mbbrigitte/Predicting_ heart_disease_UCI/blob/master/heartdisease_ $\mathrm{UCl}$. Rmd.

9. Tanaka T, Voigt MD. Decision tree analysis to stratify risk of de novo non-melanoma skin cancer following liver transplantation. Journal of cancer research and clinical oncology. 2018;144(3):607-615.

10. Paxton RJ, Zhang L, Wei C, Price D, Zhang F, Courneya KS, Kakadiaris IA. An exploratory decision tree analysis to predict physical activity compliance rates in breast cancer survivors. Ethnicity \& Health. 2017:1-13.

11. Moreno Hoyo, A. 2017. Predicting car accidents in Barcelona using a Random Forest model. Master's thesis, Universitat Politècnica de Catalunya.

12. Xiaohui J. Forecast model of road traffic accidents based on LS-SVM with grey correlation analysis. Application Research of Computers.
2016;3:038.

13. Khatri KL, Tamil LS. Early Detection of Peak Demand Days of Chronic Respiratory Diseases Emergency Department Visits Using Artificial Neural Networks. IEEE journal of biomedical and health informatics. 2018;22(1):285-290.

14. Oijuela-Canon AD, Gomez-Cajas DF, Sepulveda A. 2015. Respiratory Diseases discrimination based on acoustic lung signals and neural networks. In Signal Processing, Images and Computer Vision (STSIVA), 20th Symposium. IEEE.

15. McKinley R, Häni L, Gralla J, El-Koussy M, Bauer $S$, Arnold M, Wiest R. Fully automated stroke tissue estimation using random forest classifiers (FASTER). Journal of Cerebral Blood Flow \& Metabolism. 2017;37(8):2728-2741.

16. Chen L, Bentley P, Rueckert D. A novel framework for sub-acute stroke lesion segmentation based on random forest. Ischemic Stroke Lesion Segmentation. 2015:9.

17. Xu W, Zhang J, Zhang Q, Wei X. 2017. Risk prediction of type II diabetes based on random forest model. In Advances in Electrical, Electronics, Information, Communication and Bio-Informatics (AEEICB). Third International Conference. IEEE.

18. Shukla N, Arora M. Prediction of Diabetes Using Neural Network \& Random Forest Tree. International Journal of Computer Sciences and Engineering. 2016;4:101-104.

19. Basaia S, Agosta F, Wagner L, Magnani G, Filippi M. 2018. Automatic classification of patients with Alzheimer's disease (AD) and mild cognitive impairment $(\mathrm{MCl})$ who will convert to $A D$ using deep neural networks.

20. Lu D, Popuri K, Ding GW, Balachandar R, Beg MF. Multimodal and multiscale deep neural networks for the early diagnosis of alzheimer's disease using structural MR and FDG-PET images. Scientific Reports. 2018;8(1):5697.

21. Ravindra BV, Sriraam N, Geetha M. Classification of non-chronic and chronic kidney disease using SVM neural networks. International Journal of Engineering and Technology (UAE). 2018;7(1.3):191-194.

22. Annapoorani JCB, Gnanaselvam CR. Enhancing prediction accuracy of chronic kidney disease using neural networks. Automation and Autonomous System. 2018;10(1):10-15.

23. Wiemken TL, Furmanek SP, Mattingly WA, Guinn 
BE, Cavallazzi R, Fernandez-Botran R, Ramirez

JA. Predicting 30-day mortality in hospitalized patients with community-acquired pneumonia using statistical and machine learning approaches. The University of Louisville Journal of Respiratory Infections. 2017;1(3):10.

24. Menéndez RV. The diagnostic evaluation of rapid sputum technics for pneumococcus in community-acquired pneumonia. The usefulness of Bayes theorem for clinical application. Archivos de bronconeumologia. 1995;31(7):317322.

25. Ayat S, Farahani HA, Aghamohamadi M, Alian M, Aghamohamadi S, Kazemi Z. A comparison of artificial neural networks learning algorithms in predicting tendency for suicide. Neural Computing and Applications. 2013;23(5):13811386.

26. Bhat HS, Goldman-Mellor SJ. 2017. Predicting Adolescent Suicide Attempts with Neural Networks. arXiv preprint arXiv:1711.10057.

27. Gondi S, Patel K. Improving rural health: how system-level innovation and policy reform can enhance health outcomes across the United States. IEEE pulse. 2016;7(6):8-12.

28. Mohr DC, Zhang M, Schueller SM. Personal sensing: understanding mental health using ubiquitous sensors and machine learning. Annual Review of Clinical Psychology. 2017; 13:23-47. 